\title{
Prácticas de crianza predictoras de problemas moderados de conducta en niños
}

\author{
Silvia Morales Chainé', Danae Celeste Ramírez Arriaga', Marcela Rosas Peña', Jennifer Lira Mandujano² \\ ' Facultad de Psicología, Universidad Nacional Autónoma de México. \\ ${ }^{2}$ Facultad de Estudios Superiores Iztacala, Universidad Nacional Autónoma de México.
}

\section{RESUMEN}

Introducción: las prácticas positivas y negativas de crianza se asocian con problemas severos de conducta infantil. Objetivo: identificar las prácticas de crianza que predicen problemas moderados de conducta en niños. Método: participaron 508 padres, de 35.6 años de edad en promedio. Se utilizaron el Inventario de Prácticas de Crianza, el Cuestionario de Parentalidad de Alabama, el de Habilidades de Manejo Infantil y el Inventario de Conducta Infantil. Además, se usaron análisis descriptivos y de regresión lineal múltiple. A través del modelamiento de ecuaciones estructurales, se obtuvo un ajuste de los datos con una $X 2(1310)=1879.18 ; p=0.000, c f i=0.872$, rmsea $=0.048$, ic de 0.043 a 0.053 y un srmr $=0.076$. Las conductas características de la hiperactividad tuvieron el mayor promedio ( $m=40.6, d e=21.9)$, seguidas por las de oposición y desafío $(m=40.5 d e=22.4)$, la inatención ( $m=37.9$, de $=23.2)$ y la disrupción/agresión $(m=21.3, d e=20.1)$. El factor oposición y desafío estuvo predicho por el factor de disciplina inconsistente $(r 2=$ 0.785), la disrupción/agresión por la falta de monitoreo y supervisión $(r 2=0.723)$ y la hiperactividad por el castigo corporal $(r 2=0.590)$, en tanto que la inatención estuvo predicha inversamente por las ganancias materiales entregadas por los padres $(r 2=-0.175)$ y directamente por la disciplina inconsistente $(r 2=0.560)$. Discusión y conclusiones: las prácticas negativas de crianza como el uso del castigo corporal, la disciplina inconsistente, la entrega de ganancias materiales para mejorar la interacción con los hijos y el pobre monitoreo y supervisión predijeron de manera confiable la ocurrencia moderada de las conductas de hiperactividad, oposición, desafío, inatención, disrupción y agresión, respectivamente. El entrenamiento conductual a padres deberá incorporar prácticas positivas de crianza para la reducción de problemas moderados de conducta infantil.

\begin{abstract}
Introduction: positive and negative raising practices are associated to severe child behavioral problems. Objective: to identify the raising practices that predict children behavioral moderated problems. Method: we worked with 508 parents, which mean years old was 35.6. We used the Raising Practices Inventory, the Parenting Questionnaire-Alabama, the Children Manegement Skills one and the Child Behavior Inventory. We used descriptive and multiple lineal regression analysis. Throughouth an structured equation modeling, we obtained a data adjustment with a $X 2(1310)=1879.18 ; p=0.000, C F I=$ $0.872, R M S E A=0.048, C l$ of 0.043 , a 0.053 and a SRMR $=0.076$. The characteristical behaviors of hiperactivity had a highest mean $(M=40.6 D E=21.9)$, followed by the opositional and defiant ( $M=40.5 D E=22.4)$, innatentional $(M=37.9 D E=23.2)$ and aggresive and disruptional ones $(M=21.3 D E=20.1)$. The inconsistent discipline did predict oppositional and defiant behaviors $(r 2=0.785)$; lack of monitoring and supervision did predict disruption and aggression $(r 2=0.723)$; physical punishment did predict hiperactivity $(r 2=0.590)$; and material gains delivering $(r 2=-0.174)$ and inconsistent discipline $(r 2=0.560)$ did predict inattentional behaviors. Conclusion and discussion: negative raising practices such as physical punishment, inconsistent discipline, delivery of material gains to improve children interaction, and poor monitoring and supervision, did predict moderate ocurrence of hiperactivity, oppositional, defiant, inattentional, disruptional and aggressive behaviors, respectivily. Behavioral parent training should consider positive raising practices to reduce children moderated behavioral problems.
\end{abstract}

Keywords: raising practices, child behavior and structured equation modeling.

Palabras clave: prácticas de crianza, conducta infantil, modelo de ecuaciones estructurales.

\footnotetext{
Autor de correspondencia: smchaine@gmail.com

Fecha de recepción: 24 de julio de 2017

Fecha de aceptación: 18 de octubre de 2017

DOI: 10.28931/riiad.2017.2.04

Silvia Morales Chainé. Av. Universidad 3004, Ed. C 202, col. Copilco, del. Coyoacán, C.P. 04510, Ciudad de México. Correo electrónico: 


\section{INTRODUCCIÓN}

En México, seis de cada 10 niños se encuentran en riesgo de padecer violencia (Fondo de las Naciones Unidas para la Infancia, 2014) y con ello, de desarrollar problemas de conducta infantil. De acuerdo con el Instituto Nacional de Salud de Estados Unidos (NIH, por sus siglas en inglés, 2016), 20\% de los niños en edad escolar está en riesgo de presentar problemas de conducta que, en promedio, se inician alrededor de los ocho años de edad. En un estudio reciente realizado en México, Morales, Martínez, Nieto y Lira (2017) encontraron que 56\% de los padres reportaron problemas severos de conducta en sus hijos. Casi cuatro de cada 10 mostraron síntomas de oposición desafío, comórbidos con inatención e hiperactividad (American Psychiatric Association [APA], 2013), y uno de cada 10 presentó oposición y desafío, en exclusivo; ambos hallazgos fueron similares a los de Caraveo y Anduaga (2007). El resto de los participantes (Morales et al., 2017), además de los síntomas expuestos, reportaron en sus hijos rasgos concurrentes de conducta agresiva.

De acuerdo con Robins (1978), abordar los problemas de conducta en la infancia permitirá enfrentar de manera eficiente la progresión hacia conductas antisociales en la edad adulta. En particular, Frick y White (2008) indicaron que el desorden oposicionista desafiante, asociado con agresividad y trastorno por déficit de atención con hiperactividad, predice el inicio temprano del desorden de conducta y, posteriormente, el uso temprano de sustancias psicoactivas (Wymbs et al., 2012).

Hawes, Price y Dadds (2014) señalaron que los factores de riesgo vinculados con los problemas de conducta infantil pueden variar en su papel predictor y mediador. También indicaron que en la literatura se mencionan conductas no severas de conducta y conductas severas; estas últimas, en apariencia, son sinónimo de emociones prosociales limitadas (Rutter, 2012) y de diversidad de dificultades de conducta como oposición, desafío, agresión, hiperactividad e inatención, comórbidas entre sí (Morales et al., 2017). Comentaron, asimismo, que las variables que han sido medidas de forma consistente, a manera de informe y por observación directa, son el oposicionismo desafiante, la agresión y la inatención con o sin hiperactividad.

En concreto, la función predictiva y moderadora de los factores asociados con los problemas de conducta infantil puede estar representada por las prácticas de crianza. Aunque, en general, se ha planteado que los problemas de conducta parecen ser resultado de una disciplina rígida, inconsistente y coercitiva (Yeh, Chen, Raine, Baker, \& Jacobson, 2011).
Clark y Frick (2016), McDonald, Dodson, Rosenfield y Jouriles (2011), y Morales et al. (2017) han mencionado que las prácticas de crianza se clasifican en positivas y negativas. Sobre las primeras, Clark y Frick (2016) concluyeron que el reforzamiento positivo que usan los padres tiene una relación inversa con los problemas de conducta y que, en específico, la calidez observada en la interacción con los hijos se asocia de manera opuesta con estos mismos síntomas; ellos observaron estos fenómenos al controlar la severidad de los problemas de conducta en los análisis de resultados de los estudios que realizaron. Gryczkowski, Jordan y Mercer (2010) también reportaron que los padres de niños con problemas de conducta muestran poco involucramiento, disciplina inconsistente y falta de parentalidad positiva y monitoreo. Pardini, Fite y Burke (2008) reportaron que la falta de comunicación predice el comportamiento problemático en los niños; sin embargo, concluyeron que ni el castigo severo ni el involucramiento positivo, la poca supervisión o la disciplina inconsistente estuvieron relacionados con los problemas de conducta en niños. Por el contrario, Burke, Pardini, y Loeber (2008) sí reportaron que la falta de involucramiento positivo de los padres de familia se asocia con una mayor ocurrencia de comportamientos oposicionistas desafiantes. Encontraron, también, que existe una asociación entre el comportamiento infantil y las disciplinas tímida (Burke et al., 2008) o punitiva utilizadas por los padres (Stormshak, Bierman, McMahon, Lengua, \& Conduct Problems Prevention Research Group, 2000).

Morales et al. (2017) reportaron un mayor índice de involucramiento positivo e interacción social, como prácticas positivas, en el grupo de padres con niños sin problemas de conducta. También señalaron que la agresión infantil parece jugar un papel relevante en la asociación de las prácticas negativas (castigo corporal o inconsistencia en la disciplina) y positivas (interacción social e involucramiento positivos), con los problemas severos de conducta (también revise McDonald et al., 2011).

Con la finalidad de determinar qué prácticas de crianza de orden positivo pueden mediar la ocurrencia de problemas de conducta infantil, ciertos estudios han reportado que las instrucciones claras, el establecimiento de reglas, la solución de problemas y la interacción social positiva se asocian con la reducción de la oposición, el desafío, la agresión (Frick \& White, 2008) e, inclusive, la hiperactividad y la inatención (Morales, Félix, Rosas, López, \& Nieto, 2015).

En México, Morales y Vázquez (2014) y Morales, Martínez, Martín del Campo y Nieto (2016) reportaron un cambio en la conducta oposicionista desafiante y agresiva, a partir de la reducción del castigo, el aprendizaje de procedimientos de corrección, el uso del elogio, la 
impartición de instrucciones claras, el establecimiento de reglas, la solución de problemas y la interacción social positiva. En este sentido, ciertos estudios han indicado que los problemas graves de conducta pueden asociarse con una carencia de respuesta, por parte de los niños, al contexto asociado con la ocurrencia del castigo, en tareas en las que gradualmente se incrementan las razones del castigo a respuestas que han sido reforzadas de manera previa (por ejemplo, Frick, Ray, Thornton, \& Kahn, 2014).

Entonces, además de diferenciar entre prácticas de crianza positivas y negativas, se ha distinguido entre los problemas de conducta menores y los severos. Los últimos, son los que parecen progresar hasta la adolescencia, como resultado de estilos de crianza caracterizados por su baja calidez (Kochanska, Kim, Boldt, \& Yoon, 2013; Kroneman, Hipwell, Loeber, Koot, \& Pardini, 2011; Waller, Gardner, \& Hyde, 2013). Los problemas de conducta severos parecen asociarse con estilos de apego desorganizados (Pasalich, Dadds, Hawes, \& Brennan, 2012), en los que parece haber menos contacto visual tanto con padres como con madres en situaciones de juego libre, esto en independencia del déficit de atención con hiperactividad, los problemas de conducta que presentan (Dadds, Jambrak, Pasalich, Hawes, \& Brennan, 2010) y los bajos niveles de afecto físico y verbal con sus padres (Dadds et al., 2012). Así, las madres de niños con problemas severos de conducta, que además tenían una relación deficiente con las figuras de autoridad o carencia de culpa, han reportado menor involucramiento materno y parentalidad positiva, que las madres con niños con problemas menores de conducta (Wall, Frick, Fanti, Kimonis, \& Lordos, 2016).

En el caso de los niños con severos problemas de conducta, la oposición infantil y el bajo involucramiento positivo de los padres se ha relacionado con la agresión en ellos (Morales et al., 2017). El castigo corporal ha predicho la oposición; la inconsistencia en la disciplina, la inatención; y el castigo corporal y la inconsistencia, la hiperactividad (Morales et al., 2017); todo ello cuando hay comorbilidad entre los trastornos.

A partir de los hallazgos previos, se entiende que las prácticas de crianza implementadas durante el entrenamiento conductual a padres han sido la clave para reducir una gran diversidad de problemas de conducta (Chorpita et al., 2011; Eyberg, Nelson, \& Boggs, 2008; McMahon, 2015; Morales \& Vázquez, 2014). El entrenamiento a padres para incrementar la interacción positiva (Cartwright-Hatton et al., 2011; Cornell \& Frick, 2007), el uso de estrategias para implementar la obediencia (Forehand, Jones, \& Parent, 2013), las habilidades sociales (Haas et al., 2010), la solución de problemas (McMahon, 2015; Morales \& Vázquez, 2014; Morales et al., 2016) y el establecimiento de reglas (Morales \& Vázquez, 2014;
Morales et al., 2016) han promovido la reducción de la conducta oposicionista desafiante, la agresión, la hiperactividad y la inatención.

En el campo de la prevención universal, Morales y Vázquez (2014) encontraron que una reducción de las prácticas negativas, como el uso del castigo, además del aprendizaje de procedimientos de corrección y de prácticas positivas, como el reforzamiento positivo, redujeron los problemas moderados de conducta. Sin embargo, la presencia de conducta agresiva, junto con el desorden oposicionista desafiante o la conducta hiperactiva, parece jugar un papel relevante en la determinación de lo que constituye la severidad de los mismos (Byrd, Loeber, \& Pardini, 2012; Fritz, Wiklund, Koposov, Klinteberg, \& Ruchkin, 2008) y la efectividad de las intervenciones conductuales.

Högström, Enebrink y Ghaderi (2013) reportaron que el reforzamiento positivo y el elogio provocaron una reducción de problemas de conducta no severos, pero no encontraron un efecto importante en los niños que padecían dificultades graves. También, Manders, Dekovic, Asscher, Van Der Laan y Prins (2013) hallaron que la terapia multisistémica disminuye los problemas de conducta en niños con bajos niveles de emociones prosociales limitadas, pero no en aquellos con altos niveles de dichos rasgos. De forma contraria, Kimonis, Bagner, Linares, Blake, y Rodríguez (2014) señalaron que los niños con problemas severos de conducta parecen ser más reactivos al reforzamiento positivo que a las estrategias basadas en el castigo (disciplina), porque parecen ser menos sensibles al castigo o a sus señales.

Dadds et al. (2012) habían reportado que los niños con problemas moderados de conducta reaccionaban al entrenamiento a padres, pero que quienes padecían altos niveles de emociones prosociales limitadas mostraron un incremento de conducta oposicionista desafiante a los seis meses, con relación a su línea base. También encontraron que la combinación del entrenamiento a padres, con el de reconocimiento de emociones, produjo un cambio sostenible en la reducción de la conducta oposicionista desafiante en esta muestra de niños, pero que dicho beneficio no se presentó en los que padecían problemas moderados de conducta. Dadds et al. (2012) concluyeron que la efectividad del componente de reconocimiento de emociones no modificó esta variable en los niños, pero si incrementó la interacción familiar y la relación cálida entre sus miembros, elementos que explicaron el mantenimiento del cambio en la reducción de la conducta oposicionista desafiante.

En resumen, los hallazgos indican que los problemas severos de conducta parecen ser resultado de la carencia de interacción familiar positiva, la falta de manejo y control conductual basado en la corrección del compor- 
tamiento y el uso del castigo físico; asimismo, señalan que los problemas moderados de conducta pueden ser consecuencia del uso de prácticas de crianza que se han descrito de forma general, y que éstas pueden ser impactadas de manera efectiva por el entrenamiento a padres, quienes pueden mantener su cambio.

Uno de los objetivos de este estudio fue describir con precisión las prácticas de crianza asociadas con problemas moderados de conducta en niños, pues ello facilitará la prevención universal del consumo de drogas en etapas posteriores de la vida, a través de la aplicación exitosa de programas basados en intervenciones familiares que promueven la participación activa de los padres, el desarrollo de competencias sociales y la crianza positiva (NIH, 2016). Asimismo, se buscó identificar las prácticas de crianza que predicen problemas moderados de conducta en niños.

\section{MÉTODO}

\section{Participantes}

Se trabajó con 508 padres del sur de la Ciudad de México que reportaron tener hijos de entre dos y 12 años de edad y que expresaron su libre intención por colaborar con información sobre sus estrategias de crianza; todo ello en escuelas de nivel básico del sector público. Por lo tanto, la muestra fue de tipo intencional no probabilística por conveniencia. El promedio global de edad de los padres participantes fue de 35.6 años ( $D E=4.92)$. $85 \%$ de ellos fueron mujeres, $4 \%$ no tenía estudios, $13 \%$ tenía estudios básicos, $63 \%$ de educación media y $20 \%$ de educación media superior. 44\% de los participantes se dedicaba al hogar, 39\% era empleado y $17 \%$ comerciante. El promedio de edad de los niños, en el rango de 2 a 7 años de edad, fue de 5 años ( $D E=1.56)$; y de los niños, del rango de edad de los 7 a los 12, fue de nueve años ( $D E=1.73) .54 \%$ de los niños fueron varones y $46 \%$, niñas.

Los padres firmaron un consentimiento informado, en el que se estableció que la duración de su participación sería de una sesión; que los resultados del estudio se usarían para investigación epidemiológica y difusión de resultados; que se mantendría la confidencialidad de la información, a través de la utilización de promedios grupales; y que los padres tenían derecho a declinar el uso de su información y participación en cualquier momento del estudio, sin perjudicar su intervención en el plan de tratamiento. El estudio no otorgó incentivos, pero a los padres se les explicó el beneficio social de su participación en la implementación de estrategias efectivas para la atención psicológica. El procedimiento de trabajo fue sometido a revisión y aprobado por la Comisión de Ética de la Facultad de Psicología de la Universidad Nacional Autónoma de México.

\section{Instrumentos}

Cuestionario de Habilidades de Manejo Infantil (CHAMI; Morales \& Vázquez, 2011): autoaplicable de lápiz y papel, que consta de 11 viñetas o situaciones simuladas de evaluación sobre las habilidades de manejo de conducta infantil. Plantea situaciones hipotéticas de interacción problemática con el niño, para que los padres respondan, de manera abierta, qué harían ante dicha situación. Está integrado por cuatro subescalas: ignorar como técnica para promover conducta adecuada (ITCA); elogio; instrucciones claras, solución de problemas y establecimiento de reglas (ICSE); e interacción social y académica (ISA). Obtuvo una consistencia interna por alfa de Cronbach de 0.62 y una varianza explicada de $55 \%$, por medio de un análisis factorial exploratorio que arrojó cuatro factores. También se obtuvo la fiabilidad promedio entre evaluadores entrenados en el código de calificación, siempre mayor a 80\%.

Inventario de Prácticas de Crianza (IPC; López, 2013): cuestionario autoaplicable de lápiz y papel de 20 minutos de aplicación. Consta de 40 preguntas cerradas, que se responden en una escala de siete opciones, que van de nunca (0) hasta siempre (6), y evalúan el reporte de los padres con respecto a la disciplina y la promoción del afecto que implementan con sus hijos. El IPC tuvo una consistencia interna de 0.89 y una varianza explicada de $61.85 \%$. A través del análisis factorial exploratorio se utilizaron seis escalas: castigo (reactivos 14 al 20,33 y 38), ganancias materiales (reactivos $1,7,21,26$, 28 y 39) interacción social (reactivos 2 al 6, 9, 10, 12 y 13), normas (reactivos 29 al 32, 34 y 35), ganancias sociales (reactivos 22 al 25 y 27) y limites (reactivos 36, 37 y 40).

Inventario de Conducta Infantil (ICl; Morales \& Martínez, 2013): autoaplicable de lápiz y papel de 32 reactivos que puede resolverse en cerca de 20 minutos. En él, el padre señala el grado o intensidad con el que se presentan los comportamientos infantiles con una escala Likert de cinco puntos que va de nunca (0) a siempre (4). La consistencia interna del instrumento fue de 0.94 . El instrumento tuvo una varianza explicada de $57.32 \%$. A través de un análisis factorial exploratorio, se observó la existencia de cuatro factores: comportamiento oposicionista desafiante (reactivos 1 al 8), comportamiento disruptivo agresivo (reactivos 9, 11 y 12), inatención (reactivos 15 al 23) e hiperactividad (reactivos 24 al 32).

Cuestionario Parental Alabama (Frick, 1991): cuestionario de lápiz y papel con 42 preguntas con escala Likert de cinco opciones: nunca, casi nunca, algunas veces, frecuentemente y siempre. Aunque se reporta una con- 
sistencia interna de 0.68 en el estudio de Frick (1991), con la muestra del estudio se obtuvo una consistencia interna de 0.75 , y una varianza explicada de $60.34 \%$. A través del análisis factorial exploratorio, se utilizaron cinco escalas que, respetando su etiqueta original, se denominaron: involucramiento positivo (reactivos 1, 4, 7, $9,11,14,15,20,23$ y 26), parentalidad positiva (reactivos $2,5,13,16,18$ y 27), supervisión y monitoreo (sólo para el rango de 7 a 12 años de edad: reactivos 6,10 , $17,19,21,24,28,29,30$ y 32), disciplina inconsistente (reactivos $3,8,12,22,25$ y 31 ) y uso de castigo corporal (reactivos 33,35 y 39 ).

\section{PROCEDIMIENTO}

Se realizó un estudio correlacional causal que consistió en una sesión de evaluación escrita con formato grupal, en el que los padres recibieron los cuestionarios descritos en el apartado de instrumentos. Se dieron las siguientes instrucciones:

"En esta sesión se realizará una serie de cuestionarios que nos permitirá conocer las habilidades con las que ustedes cuentan para corregir a sus hijos en este momento y la frecuencia con la que se observan ciertas conductas en ellos. El llenado de los cuestionarios es individual, ¿tienen alguna pregunta? Podemos comenzar".

\section{Análisis estadísticos}

Con la finalidad de representar los datos se llevaron a cabo los análisis descriptivos y de regresión múltiple con el método paso a paso, para los niveles de medición intervalar, a través del paquete IBM $®$ SPSS $\otimes$ statistics versión 19. Para estimar el efecto entre las variables de estudio se especificó e identificó el modelo, se estimaron los parámetros minimizando las diferencias entre la covarianza observada y la estimada, y se evaluó el ajuste de los datos, a través de la X2, así como los índices CFI, RMSEA y SRMR para los análisis factoriales exploratorios, confirmatorios y de ecuaciones estructurales, mediante el paquete estadístico R $®$ versión 3.2.2.

\section{RESULTADOS}

A continuación, se muestran los resultados del análisis descriptivo de las prácticas de crianza de la muestra, las regresiones lineales múltiples, las variables latentes utilizadas y el modelamiento de ecuaciones estructurales de la relación entre las variables explicativas.

En la Tabla 1, se representan los puntajes promedio y las desviaciones estándar para cada una de las escalas de los instrumentos de evaluación. En el caso del ICl, el informe de conducta infantil reveló que las prácticas de crianza más recurrentes - del mayor promedio al menorfueron: hiperactividad $(M=40.6, D E=21.9)$, oposición y desafío $(M=40.5, D E=22.4)$, inatención $(M=37.9$, $D E=23.2)$ y disrupción/agresión $(M=21.3, D E=20.1)$. Obsérvese que en el caso del IPC, la práctica de crianza con el mayor promedio reportado fue la interacción social $(M=80.5, D E=13.2)$, mientras que la de menor promedio fue la entrega de ganancias materiales $(M=$ 50.7, $D E=23.5$ ). En el caso del Alabama, la práctica de

Tabla 1.

Representa los promedios y desviaciones estándar de los puntajes obtenidos en los instrumentos de evaluación por los participantes del estudio.

\begin{tabular}{|c|c|c|c|c|c|}
\hline Instrumento & $M$ & $D E$ & Instrumento & M & $D E$ \\
\hline $\mathrm{ICl}$ & & & CHAMI & & \\
\hline Hiperactividad & 40.6 & 21.9 & Elogio & 34.8 & 21 \\
\hline Oposición y desafío & 40.5 & 22.4 & ICSE & 20.3 & 16.8 \\
\hline Inatención & 37.9 & 23.2 & ITCA & 10.4 & 15.4 \\
\hline Disrupción/agresión & 21.3 & 20.1 & ISA & 7.4 & 15.2 \\
\hline IPC & & & ALABAMA & & \\
\hline Interacción social & 80.5 & 13.2 & Involucramiento & 74.8 & 13.2 \\
\hline Normas & 79.9 & 18.5 & Parentalidad positiva & 74.4 & 13.9 \\
\hline Ganancias sociales & 79.6 & 21.4 & Disciplina inconsistente & 51.9 & 15.1 \\
\hline \multirow[t]{2}{*}{ Límites } & 72.1 & 24 & Castigo corporal & 43.7 & 15 \\
\hline & & & Monitoreo/supervisión & & \\
\hline Castigo & 67.1 & 21.9 & (rango 7 a 12 años de edad) & 39.5 & 8.5 \\
\hline Ganancias materiales & 50.7 & 23.5 & & & \\
\hline
\end{tabular}


Tabla 2.

Análisis de regresión múltiple con el método paso a paso de las variables predictoras de la conducta oposicionista desafiante, disruptiva/agresiva, inatención e hiperactividad

\begin{tabular}{|c|c|c|c|c|c|c|}
\hline Conducta/Paso & Variable predictora & $\mathrm{R} 2$ & $\begin{array}{c}\text { Cambio } \\
\text { en R2 }\end{array}$ & $\begin{array}{c}\text { F del } \\
\text { cambio } \\
(\mathrm{gl})\end{array}$ & $\begin{array}{l}\text { Sig. de F } \\
\text { del } \\
\text { cambio }\end{array}$ & $\begin{array}{c}\beta \\
(p \leq .01)\end{array}$ \\
\hline \multicolumn{7}{|l|}{$\begin{array}{c}\text { Conducta } \\
\text { oposicionista } \\
\text { desafiante }\end{array}$} \\
\hline $1^{\circ}$ & Disciplina inconsistente & .282 & .284 & $\begin{array}{l}131.64 \\
(1,506)\end{array}$ & .000 & .359 \\
\hline $2^{\circ}$ & Interacción social & .307 & .028 & $\begin{array}{c}13.22 \\
(2,506)\end{array}$ & .000 & -.177 \\
\hline $3^{\circ}$ & Castigo & .349 & .044 & $\begin{array}{c}22.37 \\
(3,506)\end{array}$ & .000 & .265 \\
\hline $4^{\circ}$ & Involucramiento & .360 & .012 & $\begin{array}{c}3.30 \\
(4,506)\end{array}$ & .013 & -.121 \\
\hline \multicolumn{7}{|l|}{$\begin{array}{c}\text { Conducta } \\
\text { disruptiva/ } \\
\text { agresiva }\end{array}$} \\
\hline $1^{\circ}$ & Disciplina inconsistente & .164 & .167 & $\begin{array}{c}66.68 \\
(1,506)\end{array}$ & .000 & .256 \\
\hline $2^{\circ}$ & Castigo & .207 & .045 & $\begin{array}{c}19.06 \\
(2,506)\end{array}$ & .000 & .237 \\
\hline $3^{\circ}$ & Involucramiento & .247 & .042 & $\begin{array}{c}18.41 \\
(3,506)\end{array}$ & .000 & -.168 \\
\hline $4^{\circ}$ & Interacción social & .253 & .009 & $\begin{array}{c}3.88 \\
(4,506)\end{array}$ & .050 & -.102 \\
\hline \multicolumn{7}{|l|}{ Inatención } \\
\hline $1^{\circ}$ & Disciplina inconsistente & .160 & .163 & $\begin{array}{c}65.73 \\
(1,506)\end{array}$ & .000 & .301 \\
\hline $2^{\circ}$ & Castigo corporal & .194 & .036 & $\begin{array}{c}15.27 \\
(2,506)\end{array}$ & .000 & .216 \\
\hline \multicolumn{7}{|l|}{ Hiperactividad } \\
\hline $1^{\circ}$ & Castigo & .190 & .192 & $\begin{array}{c}80.55 \\
(1,506)\end{array}$ & .000 & .243 \\
\hline $2^{\circ}$ & Disciplina inconsistente & .244 & .056 & $\begin{array}{c}24.98 \\
(2,506)\end{array}$ & .000 & .234 \\
\hline $3^{\circ}$ & Castigo corporal & .254 & .013 & $\begin{array}{c}5.68 \\
(3,506)\end{array}$ & .018 & .143 \\
\hline
\end{tabular}


crianza de mayor promedio fue el involucramiento $(M=$ 74.8, $D E=13.2$ ) y la de menor fue el monitoreo/supervisión de los padres $(M=39.5, D E=8.5$; exclusivamente en el caso de los niños entre los 7 y 12 años de edad). En el caso del CHAMI, las prácticas de crianza, del mayor promedio reportado al menor, fueron: elogio $(M=34.8$, $D E=21.0), \operatorname{ICSE}(M=20.3, D E=16.8), \operatorname{ITCA}(M=10.4$, $D E=15.4)$ e ISA $(M=7.4, D E=15.2)$.

Los análisis de regresión mostraron que la conducta oposicionista desafiante estuvo predicha por la disciplina parental inconsistente $[F(1,506)=131.64, p<.001]$, la interacción social positiva $[F(2,506)=13.22, p<.001]$, el nivel de castigo $[F(3,506)=22.37, p<.001]$ y el involucramiento de los padres $[F(4,506)=6.30, p<.001]$ (Ver Tabla 2).

El análisis de regresión indicó que la conducta agresiva estuvo predicha por la disciplina inconsistente de los padres $[F(1,506)=66.68, p<.001]$, el nivel de castigo corporal $[F(2,507)=19.06, p<.001]$, el involucramiento parental $[F(3,506)=18.41, p<.001]$ y la interacción social $[F(4,506)=3.88, p<.001]$.

El análisis de regresión indicó que la inatención de los niños estuvo predicha por la disciplina inconsistente de los padres $[F(1,506)=65.73, p<.001]$ y el nivel de castigo corporal $[F(2,506)=15.27, p<.001]$. Por último, el análisis de regresión indicó que la hiperactividad estuvo predicha por el nivel de castigo $[F(1,506)=80.55, p<$ $.001]$, la disciplina inconsistente $[F(2,506)=24.98, p$ $<.001]$ y el castigo corporal $[F(3,506)=5.68, p<.001]$.

Para el modelamiento de ecuaciones estructurales, a partir del ANOVA de los promedios observados que resultaron significativos y del análisis factorial confirmatorio, se obtuvieron 15 variables latentes (ver Tabla 3 ): castigo [reactivos 14 (0.714), 15 (0.492), $16(0.691), 17$ (0.837), 18 (0.670), 19 (0.688), 20 (0.572), 33 (0.800) y 38 (0.770)]; ganancias materiales [reactivos 1 (0.438), 7 (0.647), 21 (0.710), 26 (0.657), $28(0.467)$ y $39(0.852)]$; interacción social [reactivos $2(0.736), 3(0.757), 4(0.650)$, 5 (0.682), 6 (0.676), 8 (0.221), 9 (0.661), 10 (0.745), $11(0.293), 12(0.695)$ y $13(0.682)$ ]; normas [reactivos 29 (0.727), 30 (0.791), 31 (0.670), 32 (0.642), 34 (0.602) y 35 (0.622)]; ganancias sociales [reactivos $22(0.703)$, 23 (0.951), 24 (0.801), 25 (0.717) y 27 (0.488)]; límites [reactivos $36(0.639), 37(0.564)$ y $40(0.814)$ ]; involucramiento positivo [reactivos 1 (0.413), 4 (0.269), 7 (0.181), 9 (0.643), 11 (0.557), 14 (0.693), 15 (0.281), 20 (0.598), $23(0.345)$ y $26(0.308)$ ]; parentalidad positiva [reactivos 2 (0.284), 5 (0.798), 13 (0.836), 16 (0.793), 18 (0.049), y 27 (0.114)]; monitoreo/supervisión [sólo para el rango de 7 a 12 años de edad: reactivos 6 (0.468), 10 (0.720), 17 (0.694), $19(0.744), 21(0.566), 29(-0.280)$ y para toda la muestra: 24 (0.392), $28(0.038), 30(0.310)$ y $32(0.255)]$; disciplina inconsistente [reactivos 3 (0.227), 8 (0.728), 12
(0.459), 22 (0.728), 25 (0.266) y 31 (0.494)]; castigo corporal [reactivos $33(0.421), 35(0.344)$ y $39(0.778)]$; oposición y desafío [reactivos $1(0.670), 2$ (0.734), 3 (0.777), 4 (0.640), 5 (0.725), $6(0.700), 7(0.586)$ y $8(0.565)]$; disrupción/agresión [reactivos $9(0.411), 11(0.767)$ y 12 (0.578)]; hiperactividad [reactivos $24(0.706), 25$ (0.737), 26 (0.799), 27 (0.317), 28 (0.545), 29 (0.465), 30 (0.464), $31(0.592)$ y $32(0.585)]$; e inatención [reactivos 15 (0.667), 16 (0.795), 17 (0.754), 18 (0.797), 19 (0.874), $20(0.727), 21(0.587), 22(0.786)$ y $23(0.628)$. Los valores de Chi cuadrada, grados de libertad, valores de probabilidad y los parámetros (CFI, RMSEA, intervalos de confianza y SRMR) que validan cada variable latente, se muestran en la Tabla 3.

Para el modelo general, el análisis factorial confirmatorio arrojó una $X 2(1310)=1923.17, p=0.000, C F I=$ $0.904 ;$ RMSEA $=0.038$, con intervalos de confianza de 0.035 a 0.042 y un $S R M R=0.070$. En la Figura 1, se representa el modelo de ecuaciones estructurales; en ella se pueden observar las cargas factoriales finales de las ganancias materiales, el monitoreo (en el caso de seis reactivos para el rango de 7 a 12 años de edad), la disciplina inconsistente, el castigo corporal, la oposición, la disrupción/agresión, la inatención y la hiperactividad y los errores estándar. En el análisis de ecuaciones estructurales se obtuvo una $X 2(1310)=1879.18 ; p=$ $0.000, C F I=0.872, R M S E A=0.048, I C$ de 0.043 a 0.053 y un $S R M R=0.076$. El factor oposición y desafío estuvo predicho por el factor de disciplina inconsistente $(r 2=$ 0.785 ; error $=0.43$ ); la disrupción/agresión, por la falta de monitoreo y supervisión $(r 2=0.723$; error $=0.23$ ); y la hiperactividad, por el castigo corporal $(r 2=0.590$; error $=0.15$ ). La inatención estuvo predicha inversamente por las ganancias materiales entregadas por los padres ( $r 2$ $=-0.174$; error $=0.07)$ y directamente por la disciplina inconsistente $(r 2=0.560$; error $=0.31)$.

\section{DISCUSIÓN Y CONCLUSIONES}

El objetivo de esta investigación fue identificar las prácticas de crianza que predicen problemas moderados de conducta en los niños. Con los participantes del estudio, como en investigaciones previas, se obtuvo el reporte de conductas con niños cuyos problemas resultaron moderados (Wall et al., 2016). Los padres señalaron síntomas característicos de los trastornos oposicionista desafiante, inatención, hiperactividad y conducta agresiva, sin alcanzar un criterio diagnóstico ni la comorbilidad entre ellos, para considerarlos problemas severos de conducta. La muestra del estudio representó un orden de incidencia de problemas moderados de conducta, similares al orden en los severos de Morales et al. (2017). Es decir, los síntomas característicos de la hiperactividad fueron 

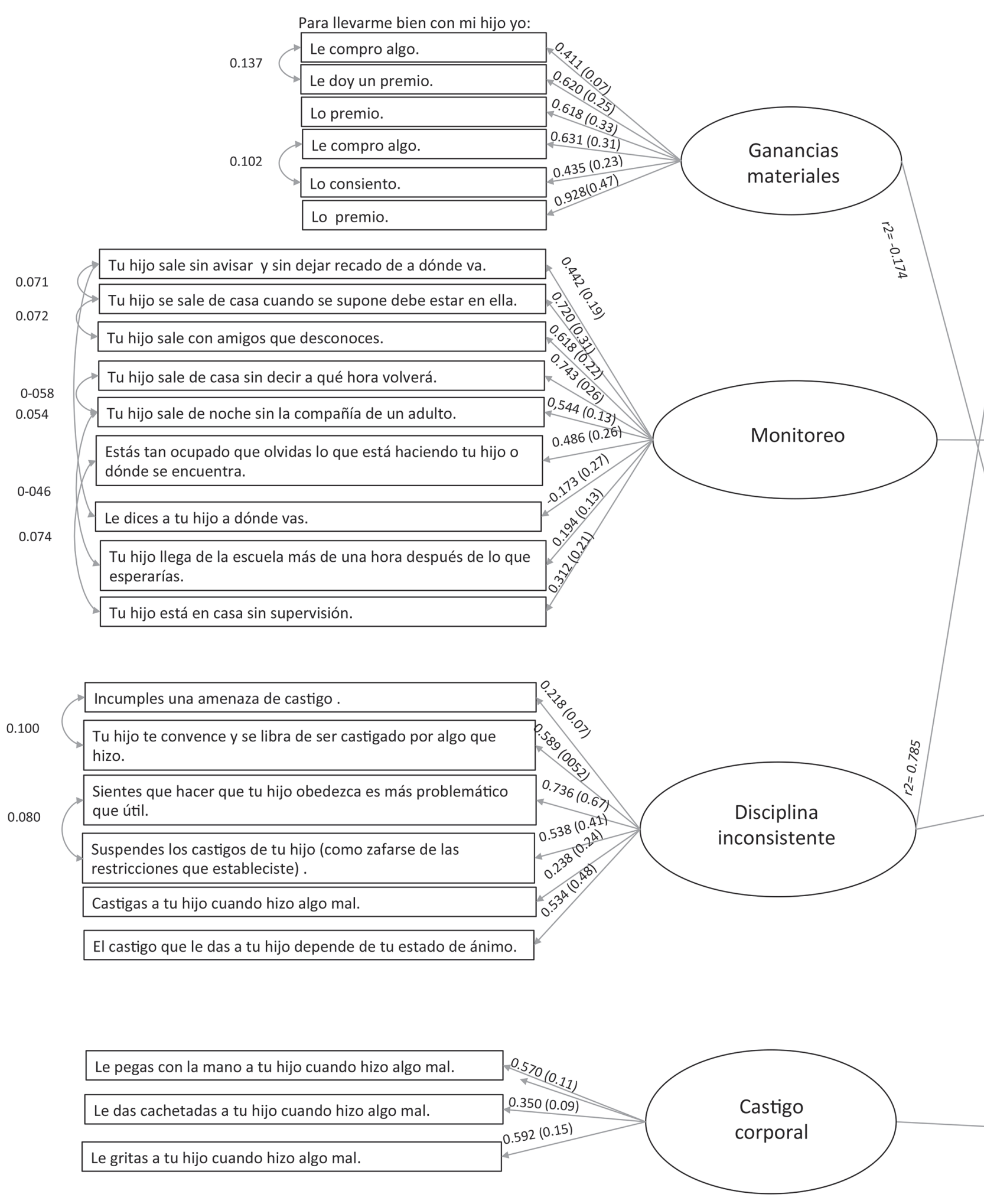

Figura 1. Modelo de ecuaciones estructurales de los problemas de conducta infantil reportados en los hijos de los participantes del estudio; y su variación en función de las prácticas de crianza relacionadas. 

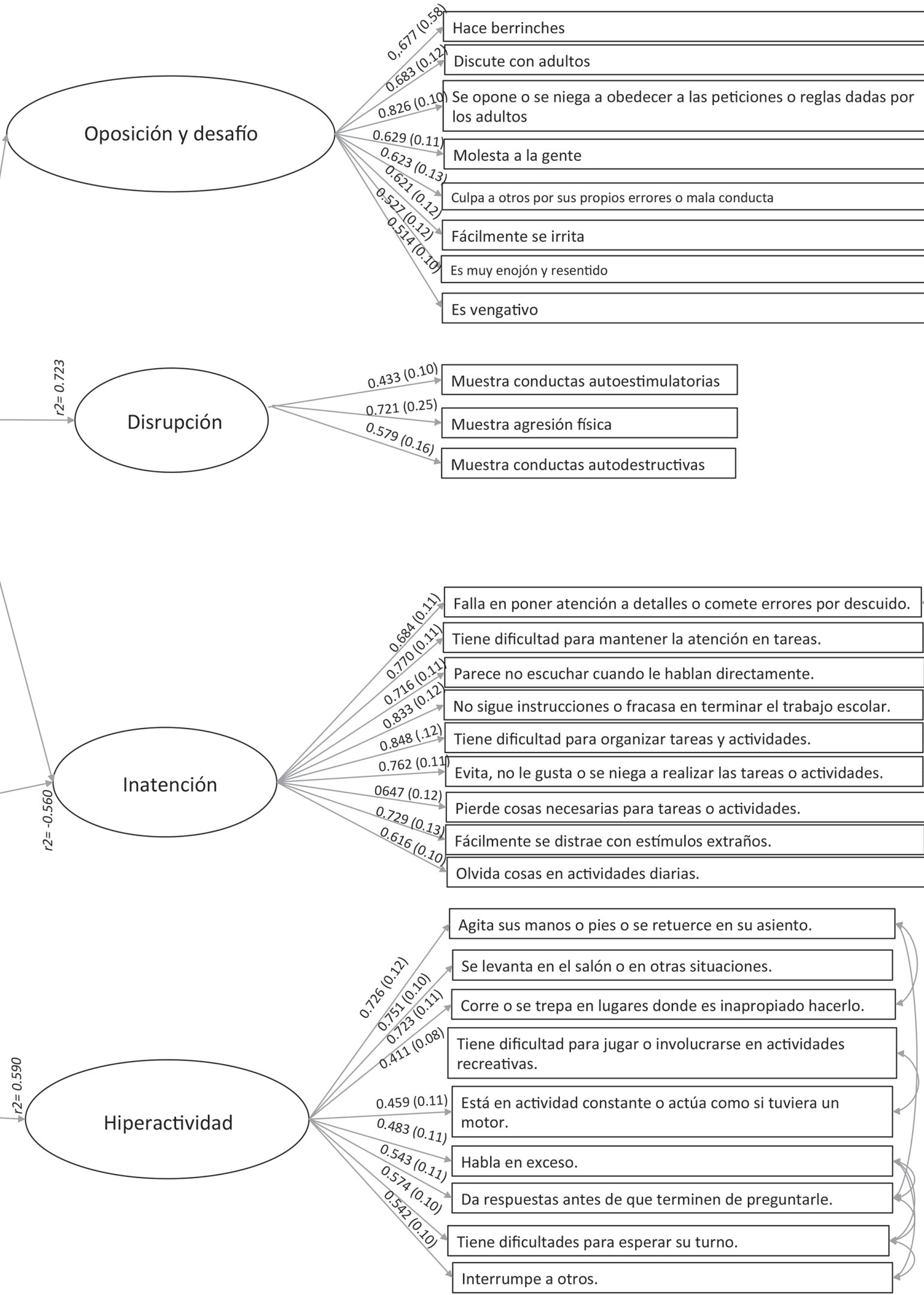
Tabla 3

Representa las variables latentes de los instrumentos de evaluación y sus grados de libertad, chi cuadrada, valor de probabilidad, CFI, RMSEA, Intervalos de confianza y SRMR.

\begin{tabular}{|c|c|c|c|c|c|c|c|c|c|}
\hline Instrumento & & Variable latente & $g l$ & $x^{2}$ & $p$ & CFI & RMSEA & $\begin{array}{c}\text { Intervalos de } \\
\text { confianza }\end{array}$ & SRMR \\
\hline \multirow[t]{6}{*}{ IPC } & 1 & Castigo & 15 & 20.51 & 0.15 & 0.998 & 0.027 & $0.000-0.053$ & 0.014 \\
\hline & 2 & Ganancias materiales & 7 & 9.52 & 0.22 & 0.996 & 0.027 & $0.000-0.065$ & 0.020 \\
\hline & 3 & Interacción social & 35 & 35.52 & 0.44 & 1.000 & 0.005 & $0.000-0.033$ & 0.019 \\
\hline & 4 & Normas & 7 & 11.32 & 0.13 & 0.994 & 0.042 & $0.000-0.085$ & 0.019 \\
\hline & 5 & Ganancias sociales & 2 & 1.43 & 0.49 & 1.000 & 0.000 & $0.000-0.096$ & 0.006 \\
\hline & 6 & Límites & 3 & 204.65 & 0 & 1.000 & 0.000 & $0.000-0.000$ & 0.000 \\
\hline \multirow[t]{5}{*}{ ALABAMA } & 7 & Involucramiento positivo & 28 & 33.04 & 0.23 & 0.991 & 0.023 & $0.000-0.049$ & 0.034 \\
\hline & 8 & Parentalidad positiva & 7 & 6.47 & 0.49 & 1.000 & 0.000 & $0.000-0.052$ & 0.018 \\
\hline & 9 & $\begin{array}{l}\text { Monitoreo y supervisión } \\
\text { (para el rango de } 7 \text { a } 12 \\
\text { años de edad) }\end{array}$ & 45 & 36.84 & 0.98 & 0.973 & 0.041 & $0.000-0.072$ & 0.041 \\
\hline & 10 & Disciplina inconsistente & 9 & 7.54 & 0.58 & 1.000 & 0.000 & $0.000-0.053$ & 0.021 \\
\hline & 11 & Castigo corporal & 3 & 95.05 & 0 & 1.000 & 0.000 & $0.000-0.000$ & 0.000 \\
\hline \multirow[t]{4}{*}{$\mathrm{ICl}$} & 12 & Oposición y desafío & 15 & 13.88 & 0.53 & 1.000 & 0.000 & $0.000-0.039$ & 0.013 \\
\hline & 13 & Disrupción/agresión & 3 & 168.2 & 0 & 1.000 & 0.000 & $0.000-0.000$ & 0.000 \\
\hline & 14 & Hiperactividad & 18 & 28.079 & 0.06 & 0.994 & 0.033 & $0.000-0.056$ & 0.026 \\
\hline & 15 & Inatención & 19 & 22.52 & 0.26 & 0.999 & 0.019 & $0.000-0.045$ & 0.016 \\
\hline
\end{tabular}

los que se presentaron con el mayor porcentaje promedio en los niños, seguidos por aquellos característicos de la oposición, el desafío, la inatención y la disrupción/ agresión, en ese orden.

También se reportaron altos índices de prácticas positivas, similares a los encontrados por Morales et al. (2015). Es decir, los padres informaron utilizar estrategias basadas en la interacción social positiva, así como usar normas y límites con sus hijos y entregar ganancias sociales por una conducta deseada; de manera adicional, hablaron sobre el involucramiento y la parentalidad positiva con sus hijos. Los niveles elevados de estas prácticas de crianza fueron similares a los reportados por Morales y Vázquez (2014), previos al entrenamiento a padres que ellos implementaron.

La incidencia de prácticas de crianza positivas, como las reportadas por los participantes del estudio, podrían determinar el nivel de moderación en la presentación de síntomas conductuales característicos de los niveles de hiperactividad, oposición, desafío, inatención, disrupción y agresividad infantil, de la misma forma que Morales et al. (2015) reportaron. Un hallazgo adicional del estudio actual fue la medición de los bajos índices de prácticas como el monitoreo/supervisión. Es decir, los índices de monitoreo y supervisión resultaron bajos y con ello bajaron también los reportes de cercanía, la verificación de las actividades de los hijos, la vigilancia de sus relaciones sociales y la permanencia dentro del hogar (Frick, 2016).

Otro conjunto de prácticas positivas de crianza que se evaluaron y resultaron en un bajo índice de ocurrencia fueron: el elogio a la conducta deseada de los hijos, la impartición de instrucciones claras para promover la obediencia infantil (Forehand et al., 2013), la solución de problemas en la familia, el establecimiento de reglas para la prevención de la conducta problemática, el empleo de la técnica de ignorar, como estrategia de cambio conductual y las conductas de interacción positiva durante la actividad académica. En el estudio de Morales et al. (2015), estos bajos índices de prácticas positivas de crianza se asociaban con conducta oposicionista desafiante y agresiva en niños; de hecho, las prácticas positivas se incrementaron como efecto del entrenamiento a padres, lo que predijo, en estudios posteriores (Morales et al., 2016), una reducción de dichos comportamientos.

De forma adicional, los padres participantes en el estudio actual informaron utilizar índices intermedios de prácticas negativas como el castigo en general, bajos niveles de castigo corporal, la entrega de ganancias 
materiales y el uso de una disciplia inconsistente. Tales resultados son congruentes con los reportes de Cornell y Frick (2007), y Frick (2016), con respecto a que el uso de tales prácticas negativas de crianza se asocian con problemas moderados de conducta en niños.

Así, en la evaluación del nivel de asociación entre la ocurrencia de prácticas positivas y negativas de crianza con problemas moderados de conducta, los hallazgos del estudio indicaron una relación múltiple relevante entre ellos. En cuanto al uso de las prácticas negativas, el informe de disciplina inconsistente y de castigo, se asoció con la ocurrencia de conductas oposicionistas desafiantes y agresivas, de la misma forma en que lo reportaron Stormshak et al., (2000) y Ayala Pedroza, Morales, Chaparro y Barragán (2002). De forma adicional, la interacción social positiva y el involucramiento de los padres, como estrategias positivas de baja incidencia, variaron a la par de la conducta oposicionista desafiante y la agresiva, como Burke et al. (2008), Gryczkowski et al. (2010) o, más recientemente, Morales et al. (2017) reportaron. Cabe resaltar que, en el caso de los síntomas de inatención e hiperactividad, las prácticas de crianza que se asociaron con ellos fueron exclusivamente negativas; esto es, la disciplina inconsistente de los padres y el nivel de castigo solo y corporal variaron con la conducta de inatención y de hiperactividad, como lo habían reportado Stormshak et al. (2000).

Otro de los hallazgos relevantes del estudio fue la ratificación de los conceptos abordados en el mismo para la valoración de los niveles predictivos entre las variables. Es decir, la capacidad de medir confiablemente los niveles de castigo, la entrega de ganancias materiales, la interacción social positiva entre padres e hijos, la impartición de normas, el uso de ganancias sociales, el establecimiento de límites, el involucramiento positivo entre la diada, la parentalidad positiva, el monitoreo y la supervisión, la inconsistencia en la disciplina, el uso del castigo corporal, la ocurrencia de conductas de oposición y desafío, la disrupción/agresión, la hiperactividad y la inatención, todo ello con la muestra del estudio, lo que permite la validación de los resultados finales. Así, fue posible evaluar el nivel predictivo de las prácticas de crianza, de clasificación negativa (Clark \& Frick, 2016, McDonald et al., 2011; Morales et al., 2017) sobre la conducta moderadamente problemática reportada por los padres (Hawes et al., 2014).

En primer término, los hallazgos indicaron que la conducta moderada de orden oposicionista desafiante es, en alto grado, consecuencia de la práctica negativa denominada disciplina inconsistente, tal como Yeh et al. (2011) habían demostrado. En específico, que los niños hagan berrinches, discutan con adultos, se opongan a obedecer, molesten a las personas, culpen a otros por lo que han hecho, se irriten con facilidad, se enojen y sean vengativos, ocurre, en mayor grado, cuando los padres usan el castigo, incumplen las amenazas de ejercerlo, se dejan convencer por sus hijos para librarse del mismo, los suspenden o la sanción depende del estado de ánimo de los padres, quienes reportan que hacer que sus hijos obedezcan es más problemático que útil (Morales et al., 2017).

En segundo término, los resultados indicaron que la conducta disruptiva y agresiva ocurrió, de forma parcial, en función de los bajos niveles de monitoreo y supervisión de los padres. Es decir que conductas en los niños, como la agresión física, variaron en función de hechos como que los niños permanezcan en casa sin supervisión o que los padres estén tan ocupados que olviden avisar a sus hijos a dónde van o desconozcan lo que sus hijos están haciendo o dónde se encuentran; todo esto, de forma similar a lo reportado por Frick en 2016.

En tercer término, los hallazgos indicaron que los síntomas menores relacionados con la hiperactividad ocurrieron, de forma parcial, en función del uso del castigo corporal (Frick et al., 2014; Morales et al., 2017; Stormshak et al., 2000), y que las conductas características de la inatención infantil ocurrieron, parcial e inversamente, por la entrega de ganancias materiales por parte de los padres y, de forma directa, por el uso inconsistente de la disciplina, según se había reportado antes (Gryczkowski et al., 2010; Morales et al., 2017; Wall et al., 2016; Yeh et al., 2011).

De acuerdo con Hawes et al. (2014), la incidencia de reportes de conducta problemática moderada en los niños puede considerarse como un sinónimo de la ausencia de emociones prosociales limitadas en los participantes de este estudio. Con tal consideración, vale la pena indicar la necesidad de orientar los hallazgos del actual estudio a valorar si las prácticas negativas de crianza, como el uso del castigo corporal, la disciplina inconsistente, la entrega de ganancias materiales, el pobre monitoreo o la baja supervisión, predicen de manera confiable la baja incidencia de emociones prosociales limitadas, como las define Frick (2016); o si la promoción de prácticas positivas, como la interacción social en la familia, el elogio o el involucramiento parental, permiten resolver, con mayor eficacia, la ocurrencia de altos índices de emociones prosociales, limitadas en niños mexicanos, al igual que en niños de otras culturas en el mundo.

Estudios adicionales deberán considerar el rango de ocurrencia de las emociones prosociales limitadas, desde la baja o moderada ocurrencia de conductas problema hasta la severa para definirlas operacionalmente. Asimismo, a partir del entrenamiento a padres deberá analizarse si las prácticas de crianza positivas y negativas permiten modular la ocurrencia de emociones pro- 
sociales limitadas o, dicho de manera más operacional, la ocurrencia de conductas problemáticas infantilesdesde moderadas hasta severas; para ello puede ser viable considerar la promoción de prácticas positivas de crianza, a fin de prevenir el comportamiento antisocial en la adolescencia o el consumo de drogas en etapas posteriores de la vida.

En conclusión, las prácticas negativas de crianza, como el uso del castigo corporal, la disciplina inconsistente, la entrega de ganancias materiales para mejorar la interacción con los hijos y el pobre monitoreo y supervisión, predijeron de forma confiable la ocurrencia moderada de conductas como hiperactividad, oposición, desafío, inatención, disrupción y agresión. La interacción social positiva y el involucramiento de los padres fueron prácticas que variaron, junto con las conductas oposicionistas, desafiantes y agresivas, en función de algún factor mediador no detectado en el estudio. Tales hallazgos parecen indicar que el uso del entrenamiento conductual a padres deberá, en lo sucesivo, jugar el papel de mediador de aquellas prácticas que sólo se relacionaron y variaron junto con los problemas moderados de conducta (Dadds et al., 2012; Morales \& Vázquez, 2014).

En el caso particular de padres que reportaron problemas moderados de conducta, los profesionales de la salud que imparten el entrenamiento conductual a padres pueden dirigir sus esfuerzos a reducir el uso del castigo corporal, a promover el uso de una disciplina consistente, a reducir el uso de ganancias materiales y a incrementar los niveles de monitoreo y supervisión. Estudios previos han indicado que con niños con problemas moderados de conducta, tales componentes pueden asegurar la reducción de la conducta oposicionista desafiante y agresiva, la inatención y la hiperactividad (Dadds et al., 2012; Morales \& Vázquez, 2014). Con base en las prácticas que no predijeron, pero sí se asociaron, se entiende que en el entrenamiento a padres, los profesionales de la salud pueden abordar, en segundo término, componentes como la interacción social positiva y el involucramiento (Cartwright-Hatton et al., 2011; Cornell \& Frick, 2007).

En el estudio no se observaron efectos predictivos ni asociativos de otras prácticas de crianza como el uso de normas y límites y la entrega de ganancias sociales por conducta deseada (a pesar de su alta incidencia). No obstante, se sugiere incorporar a los programas de entrenamiento, dirigido a padres de niños con moderados y severos problemas de conducta, el elogio de la conducta deseada, la impartición de instrucciones claras para promover la obediencia infantil, el uso de solución de problemas en la familia, el establecimiento de reglas para la prevención de la conducta problema, el empleo de la técnica de ignorar, como estrategia de cambio conductual, y las conductas de interacción positiva durante la actividad académica.

La presencia de tales prácticas en la muestra de estudio fue muy baja y, quizá por ello, carente de asociación con los problemas moderados de conducta infantil, pero no con los severos de conducta en otro estudios (por ejemplo, Morales et al., 2017) y con el mantenimiento del cambio conductual de problemas moderados de conducta en niños. Un tercer componente que debe considerarse en los entrenamientos conductuales a padres consiste en estrategias para implementar la obediencia (Forehand et al., 2013), las habilidades sociales (Haas et al., 2011), la solución de problemas (McMahon, 2015; Morales \& Vázquez, 2014; Morales et al., 2016), el establecimiento de reglas (Dadds et al., 2012; Morales \& Vázquez, 2014; Morales et al., 2016) y el uso del elogio (Högström et al., 2013). Todas estas estrategias, sumadas a la reducción del uso de prácticas negativas de crianza, promueven el mantenimiento en la reducción de problemas de conducta no severos (Manders et al., 2013; Morales \& Vázquez, 2014).

En México, ampliar el uso de las prácticas positivas puede funcionar como un factor protector de la salud mental en los niños. A nivel cultural, sería fundamental verificar cuáles son las barreras potenciales de la adopción de las prácticas positivas de crianza o, bien, cuáles son los catalizadores que fomentan las prácticas negativas e impiden la reducción de problemas de conducta infantil como la hiperactividad, la inatención, la oposición o la disrupción en los escenarios escolares, familiares y comunitarios.

Futuras investigaciones continuarán mostrando la relevancia y el orden de impartición de los componentes del entrenamiento conductual a padres, a fin de contribuir en el desarrollo de un programa de atención costo-efectivo para las instituciones de salud, que ayude a la reducción mantenida de los problemas moderados de conducta y a la promoción de la salud mental infantil. Con hallazgos en este sentido, será posible evaluar y prevenir la progresión de los problemas moderados de conducta hacia los desórdenes oposicionista desafiante, de agresión física impuesta por los niños y de déficit de atención con hiperactividad.

La reducción de problemas de conducta infantil constituye un campo de prevención de la conducta antisocial en la adolescencia y con ello del comportamiento adictivo en esa etapa de la vida (Frick \& White, 2008). Conforme los hallazgos de Wymbs et al. (2012), los adolescentes con desórdenes severos de conducta se encuentran en riesgo de usar mariguana y alcohol; por lo tanto, la reducción de la conducta problemática infantil, en función de las prácticas de crianza, constituye una estrategia de prevención universal en adicciones. 


\section{FUENTES DE FINANCIAMIENTO}

El presente trabajo se realizó con apoyo financiero del Proyecto PAPIIT IT300316 "Prácticas de crianza en función de las recomendaciones en video, mensajes cortos de texto y curso a distancia", otorgado por la Dirección General de Asuntos de Personal Académico (DGAPA) de la Universidad Nacional Autónoma de México (UNAM) a la responsable del proyecto, doctora Silvia Morales Chainé.

\section{CONFLICTOS DE INTERÉS}

Los autores declaran no tener conflictos de interés.

\section{REFERENCIAS}

American Psychiatric Association (2013). Diagnostic and statistical manual of mental disorders (5th ed.). Washington, DC: Autor.

Ayala, V., H., Pedroza, C., F., Morales, C. S., Chaparro, C. L. A., \& Barragán, T., N. (2002). Factores de riesgo, factores protectores y generalización del comportamiento agresivo en una muestra de niños en edad escolar. Salud Mental, 25(3), 27-40.

Burke, J. D., Pardini, D. A., \& Loeber, R. (2008). Reciprocal relationships between parenting behavior and disruptive psychopathology from childhood through adolescence. Journal of Abnormal Child Psychology, 36(5), 679-692. doi: 10.1007/S10802-0089219-7

Byrd, A. L., Loeber, R., \& Pardini, D. A. (2012). Understanding desisting and persisting forms of delinquency: the unique contributions of disruptive behavior disorders and interpersonal caIlousness. Journal of Child Psychology and Psychiatry, 53(4), 371-380. doi: 10.1111/j.1469-7610.2011.02504.x

Caraveo y Anduaga, J. J., (2007). Cuestionario breve de tamizaje y diagnóstico de problemas de salud mental en niños y adolescentes: algoritmos para síndromes y su prevalencia en la Ciudad de México (Segunda parte). Salud Mental, 30(1), 48-55.

Cartwright-Hatton, S., McNally, D. Field, A. P., Rust, S., Laskey, B., \& Woodham, A. (2011). A new parenting-based group intervention for young anxious children: results of a randomized controlled trial. Journal of the American Academy of Child and Adolescent Psychiatry, 50(3), 242-251. e6. doi:10.1016/j.jaac.2010.12.015

Chorpita, B. F., Daleiden, E. L., Ebesutani, C., Young, J., Becker, K. D., Nakamura, B. J., ... Satarace, N. (2011). Evidence-based treatments for children and adolescents: an updated review of indicators of efficacy and effectiveness. Clinical Psychology: Science and Practice, 18(2), 155-172. doi: 10.1111/j.14682850.2011.01247.x

Clark, J. E. \& Frick, P. J. (2016). Positive parenting and callous-unemotional traits: their association with school behavior problems in young children. Journal of Clinical Child and Adolescent Psychology, OO(00), 1-13. doi:10.1080/15374416.2016.1253016

Cornell, A. H., \& Frick, P. J. (2007).The moderating effects of parenting styles in the association between behavioral inhibition and parent-informed guilt and empathy in preschool children. Journal of Clinical Child and Adolescent Psychology, 36(3), 305-318. doi: 10.1080/15374410701444181

Dadds, M. R., Allen, J. L., Oliver, B. R., Faulkner, N., Legge, K., Moul, C., ... Scott, S. (2012). Love, eye contact, and the developmental origins of empathy v. psychopathy. The British Journal of Psychiatry, 200(3), 191-196. doi:10.1192/bjp.bp.110.085720

Dadds, M. R., Jambrak, J., Pasalich, D., Hawes, D. J., \& Brennan, J. (2010). Impaired attention to the eyes of attachment figures and the developmental origins of psychopathy. Journal of Child Psychology and Psychiatry, 52(3), 238-245. doi:10.1111/j.14697610.2010.02323.x

Eyberg, S. M., Nelson, M. M., \& Boggs, S. R. (2008). Evidence-based psychosocial treatments for children and adolescents with disruptive behavior. Journal of Clinical Child and Adolescent Psychology, 37(1), 215-237. doi:10.1080/15374410701820117

Fondo de las Naciones Unidas para la Infancia. (2014). México. Documento del programa del país 2014-2018. Recuperado de https://www.unicef.org/about/execboard/files/2013-PL15Mexico_CPD-final_approved-Spanish.pdf

Forehand, R., Jones, D. J., \& Parent, B. A. J. (2013). Behavioral parenting interventions for child disruptive behaviors and anxiety: What's different and what's the same? Clinical Psychology Review, 33(1), 133-145. doi:10.1016/j.cpr.2012.10.010

Frick, P. J. (1991). The Alabama Parenting Questionnaire. Unpublished rating scale. Alabama: University of Alabama.

Frick, P. J. (2016). Early identification and treatment of antisocial behavior. Pediatric Clinics of North America, 63(5), 861-71. doi:10.1016/j.pcl.2016.06.008

Frick, P. J., Ray, J. V., Thornton, L. C., \& Kahn, R. E. (2014). Can callous-unemotional traits enhance the understanding, diagnosis and treatment of serious conduct problems in children and adolescents? a comprehensive review. Psychological Bulletin, 140(1), 1-57. doi: 10.1037/a0033076

Frick, P. J., \& White, S. F. (2008). Research review: the importance of callous-unemotional traits for development models of aggressive and antisocial behavior. The Journal of Child Psychology and Psychiatry, 49(4), 359-375. doi: 10.1111/j.14697610.2007.01862.x

Fritz, M. V., Wiklund, G., Koposov, R. A., Klinteberg, B., \& Ruchkin, V. V. (2008). Psychopathy and violence in juvenile delinquents: what are the associated factors? International Journal of Law and Psychiatry, 31(3), 272-279. doi:10.1016/j.jilp.2008.04.010

Gryczkowski, M., Jordan, S. S., \& Mercer, S. (2010). Differential relations between mothers' and fathers' parenting practices and child externalizing behavior. Journal of Child and Family Studies, 19(5), 539-546. doi:10.1007/s10826-009-9326-2

Haas, S. M., Waschbusch, D. A., Pelham, W. E., Kings, S., Andrade, B. F., \& Carrey, N. J. (2010). Treatment response in CP/ADHD children with callous/unemotional traits. Journal of Abnormal Child Psychology, 39(4), 541-552. doi:10.1007/s10802-010-9480-4

Hawes, D. J., Price, M. J., \& Dadds, M. R. (2014). Callous-Unemotional traits and the treatment of conduct problems in child- 
hood and adolescence: a comprehensive review. Clinical Child and Family Psychology Review, 17(3), 248-267. doi: 10.1007/ s10567-014-0167-1

Högström, J., Enebrink, P., \& Ghaderi, A. (2013). The moderating role of child callous-unemotional traits in an internet-based parent-management training program. Journal of Family Psychology, 27(2), 314-323. doi: 10.1037/a0031883

Kimonis, E. R., Bagner, D. M., Linares, D., Blake, C. A., \& Rodríguez, G. (2014). Parent training outcomes among young children with callous-unemotional conduct problems with or at risk for developmental delay. Journal of Child and Family Studies, 23(2), 437448

Kochanska, G., Kim, S., Boldt, L. J., \& Yoon, J. E. (2013). Children's callous-unemotional traits moderate links between their positive relationships with parents at preschool age and externalizing behavior problems at early school age. Journal of Child Psychology and Psychiatry, 54(11), 1251-1260. doi: 10.1111/ jcpp.12084

Kroneman, L. M., Hipwell, A. E., Loeber, R., Koot, H. M., \& Pardini, D. A. (2011). Contextual risk factors as predictors of disruptive behavior disorder trajectories in girls: the moderating effect of callous-unemotional features. Journal of Child Psychology and Psychiatry, 52(2), 167-175. doi:10.1111/j.1469-7610.2010. 02300.x

López, C. F. (2013). Inventario de prácticas de crianza. En C. S. Morales, \& R. M. J. Martínez (Dirs.). Prevención de las conductas adictivas a través de la atención del comportamiento infantil para la crianza positiva. Manual del Terapeuta (pp. 14-19). México: CENADIC-SSA.

Manders, W. A., Dekovic, M., Asscher, J. J., Van Der Laan, P. H., \& Prins, P. J. (2013). Psychopathy as predictor and moderator of multisystemic therapy outcomes among adolescents treated for antisocial behavior. Journal of Abnormal Child Psychology, 41(7), 1-12. doi:10.1007/s10802-013-9749-5

McDonald, R., Dodson, M. C., Rosenfield, D., \& Jouriles, E. N. (2011). Effects of a parenting intervention on features of psychopathy in children. Journal of Abnormal Child Psychology, 39(7), 1013-1023. doi:10.1007/s10802-011-9512-8

McMahon, R. J. (2015). Parent management training interventions for preschool-age children. Parenting skills. 1-8. Ottawa: Simon Fraser University and Child \& Family Research Institute.

Morales, C. S., Félix, R. V., Rosas, P. M., López, C. F., \& Nieto, G. J. (2015). Prácticas de crianza asociadas al comportamiento negativista desafiante y de agresión infantil. Avances en Psicología Latinoamericana, 33(1), 57-76. doi: 10.12804/apl33.01.2015.05

Morales, C. S., \& Martínez, R. M. J. (2013). Prevención de las conductas adictivas a través de la atención del comportamiento infantil para la crianza positiva. Manual del terapeuta. México: CENADIC-SSA

Morales, C. S., Martínez, R. M. J., Martín del Campo, S. R., \& Nieto, G. J. (2016). Las prácticas de crianza y la reducción de los problemas de conducta infantil. Psicología Conductual, 24(2), 341-357.
Morales, C. S., Martínez, R. M. J., Nieto, G. J., \& Lira, M. J. (2017). Crianza positiva y negativa asociada con los problemas severos de conducta infantil. Salud y Drogas, 17(2), 137-149.

Morales, C. S., \& Vázquez, P. F. (2011). Evaluación de conocimientos sobre habilidades de manejo conductual infantil en profesionales de la salud. Acta de Investigación Psicológica, 1, 428-440.

Morales, C. S., \& Vázquez, P. F. (2014). Prácticas de crianza asociadas a la reducción de los problemas de conducta infantil: una aportación a la salud pública. Acta de Investigación Psicológica, 4, 1700-1715.

National Institute of Health. (2016). Trastorno de oposición desafiante. Estados Unidos: Medline Plus. Recuperado de https://medlineplus.gov/spanish/ency/article/001537.htm

Pardini, D. A., Fite, P. J., \& Burke, J. D. (2008). Bidirectional associations between parenting practices and conduct problems in boys from childhood to adolescence: the moderating effect of age and African American ethnicity. Journal of Abnormal Child Psychology, 36(5), 647-663. doi:10.1007/s10802-007-9162-z

Pasalich, D. S., Dadds, M. R., Hawes, D. J., \& Brennan, J. (2012). Attachment and callous-unemotional traits in children with early-onset conduct problems. Journal of Child Psychology and Psychiatry, 53(8), 838-845. doi:10.1111/j.1469-7610.2012.02544.x

Robins, L. N. (1978). Study childhood predictors of adults antisocial behavior: replications from longitudinal studies. Psychological Medicine, 8(04), 611-622. doi:10.1017/s0033291700018821

Rutter, M. (2012). Psychopathy in childhood: is it a meaningful diagnosis? The British Journal of Psychiatry, 200(3), 175-176. doi:10.1192/bjp.bp.111.092072

Stormshak, E. A., Bierman, K. L., McMahon, R. J., Lengua, L. J., \& Conduct Problems Prevention Research Group. (2000). Parenting practices and child disruptive behavior problems in early elementary school. Journal of Clinical Child Psychology, 29(1), 17-29. doi: 10.1207/s15374424jccp2901_3

Wall, T. D., Frick, P. J., Fanti, K. A., Kimonis, E. R., \& Lordos, A. (2016). Factors differentiating callous-unemotional children with and without conduct problems. Journal of Child Psychology and Psychiatry, 57(8), 976-83. doi: 10.1111/jcpp.12569

Waller, R., Gardner, F., \& Hyde, L. W. (2013). What are the associations between parenting, callous-unemotional traits, and antisocial behavior in youth? A systematic review of evidence. Clinical Psycology Review, 33(4), 593-608. doi: 10.1016/j. cpr.2013.03.001

Wymbs, B. T., McCarty, C. A., King, K. M., McCauley, E., Stoep, A. V., Baer, J. S., ... Waschbusch, D. A. (2012). Callous-unemotional traits as unique prospective risk factors for substance use in early adolescent boys and girls. Journal of Abnormal Child Psychology, 4O(7), 1099-1110. doi: 10.1007/s10802-012-9628-5

Yeh, M. T., Chen, P., Raine, A., Baker, L. A., \& Jacobson, K. C. (2011). Child psychopathic traits moderate relationships between parental affect and child aggression. Journal of the American Academy of Child \& Adolescent Psychiatry, 50(10), 10541064. doi: 10.1016/j.jaac.2011.06.013 